\title{
Mn and Fe ions and oxo clusters in ZSM-5: pushing the limits of X-ray spectroscopy
}

\author{
D. C. Radu ${ }^{\text {a }}$, P. Glatzel ${ }^{\text {b }}$ W. M. Heijboer ${ }^{\text {a }}$, J. H. Bitter ${ }^{\text {a }}$, B. M. Weckhuysen ${ }^{\text {a }}$ and F. M. F. \\ de Groot ${ }^{\mathrm{a}}$ \\ ${ }^{a}$ Inorganic Chemistry and Catalysis, Department of Chemistry, Utrecht University, \\ Sorbonnelaan 16, 3508 TB Utrecht, The Netherlands \\ ${ }^{\mathrm{b}}$ ESRF, 6 Rue Jules Horowitz, 38043 Grenoble, France
}

\begin{abstract}
$\mathrm{X}$-ray spectroscopic techniques are important tools for the characterization of metal ions, clusters and nanoparticles in zeolites. A major limitation in X-ray absorption spectra is the intrinsic broadening due to core hole lifetime effects. Using high-energy resolution fluorescence detection (HERFD), this intrinsic broadening is removed, revealing new fine structure. In addition, we will show that the use of circular polarized X-rays can be a useful tool to determine the aggregation of metal ions. We present results obtained using these novel X-ray spectroscopic techniques, using Fe-ZSM-5 and Mn-ZSM-5 systems as showcases. The prospects for these techniques to become "standard" tools for catalysis's research will be discussed.
\end{abstract}

\section{INTRODUCTION}

Zeolites containing metal ions or nanoparticles present an important range of catalytic systems. Although many catalysts are reported to be active in some interesting reactions, the natures of their active sites are still unknown or very difficult to prove. The challenge to identify the active species lies in the diversity of the species present in the zeolite host, so discrimination between spectator and active species is necessary. In order to achieve this, new and/or improved characterization techniques are necessary. We will present here results obtained using new concepts in X-ray and electron spectroscopic techniques, including X-ray Magnetic Circular Dichroism (X-MCD), HERFD-XANES and scanning transmission electron microscopy - electron energy loss spectroscopy (STEM-EELS) using Fe-ZSM-5 and MnZSM-5 samples as test systems.

\section{EXPERIMENTAL SECTION}

The preparation of the samples have been discussed in detail elsewhere [1,2]. The HERFDXANES measurements have been performed at the BioCAT beamline of the APS (Argonne, USA) and beamlines ID16 and ID26 at ESRF (Grenoble, France). High-resolution fluorescence spectra were measured using spherically bent Si or Ge crystals as the emission monochromator. The experimental details and beamline settings can be found elsewhere [1-3]. 
The X-MCD measurements were conducted on beamline 4.0.2. at the ALS (Berkeley, USA) [1].

\section{RESULTS AND DISCUSSION}

\subsection{HERFD-XANES}

HERFD-XANES makes use of the coherence between the X-ray absorption and emission processes. In K edge X-ray absorption, a photon of known energy will be absorbed giving rise to an electron release from the $1 \mathrm{~s}$ core shell. Due to the short lifetime of the $1 \mathrm{~s}$ core hole, another electron from a higher shell will decay giving rise to X-ray emission lines. One can tune a detector at the desired energy to measure discriminately the signal given by a particulate emission line, where the $\sim 1.5 \mathrm{eV}$ broadening (characteristic for Fe/Mn) of the deep $1 \mathrm{~s}$ core hole is replaced by the $\sim 0.4 \mathrm{eV}$ broadening of a shallow core hole, convoluted with the experimental broadenings [3]. One of the advantages of this technique is the improved separation of the pre-edge region from the edge jump region, making it possible to perform a detailed analysis of this region. Using the protocol analysis proposed by Wilke et al.[4] for Fe-containing compounds one can create a structure map between the pre-edge characteristics (i.e. the centroid position and integrated pre-edge intensity) of references compounds well characterized by crystallographic means. Comparing the pre-edge characteristics from the real in-situ data with the references can give valuable information on the local coordination and valence of the active center [1].

HERFD-XANES is applied to Mn-ZSM-5 in a $\mathrm{N}_{2} \mathrm{O}$ decomposition reaction coupled with NO re-oxidation. Details of the reaction conditions and catalyst preparations are provided elsewhere [2]. Fig. 1A shows the pre-edge region of Mn-ZSM-5 as measured with HERFDXANES. Using Charge Transfer Multiplet (CTM) calculations [3,6] the peak at $6540 \mathrm{eV}$ can be assigned to $\mathrm{Mn}^{2+}$, while the feature at $6542 \mathrm{eV}$ is due to $\mathrm{Mn}^{3+}$. The CTM pre-edge structure of $\mathrm{Mn}^{2+}$ systems contains essentially a single feature, while $\mathrm{Mn}^{3+}$ systems have a broad pre-edge that extends to below the $\mathrm{Mn}^{2+}$ peak due to the large multiplet effect of the $1 \mathrm{~s}^{1} 3 \mathrm{~d}^{5}$ final states.
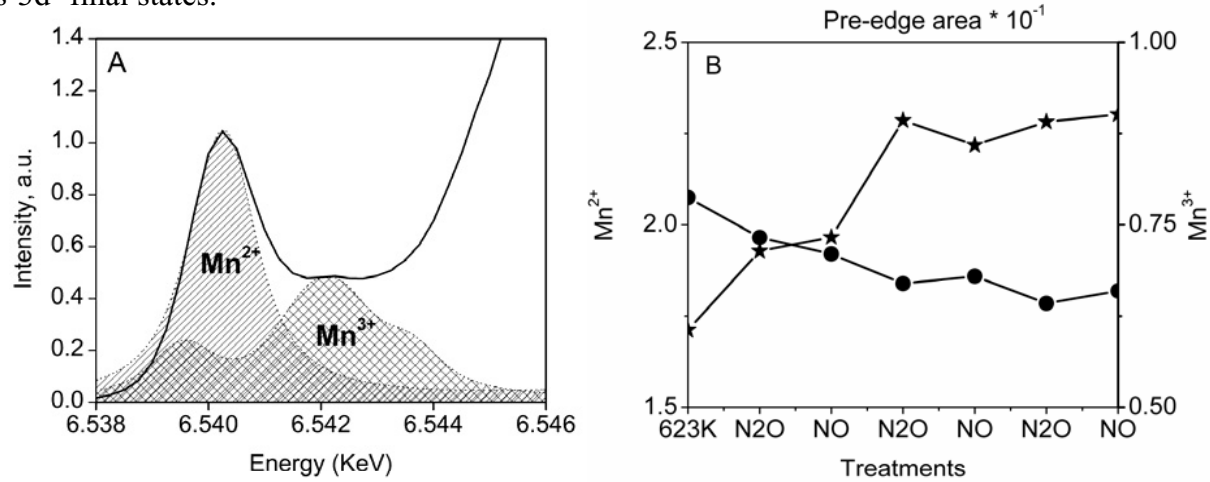

Fig. 1. (A) Components of the pre-edge region measured using HERFD-XANES technique; (B) Evolution of the pre-edge components during catalytic testing $\left(-\bullet-\right.$ for $\mathrm{Mn}^{2+}$ form and -*- for $\mathrm{Mn}^{3+}$ form) 
Fig. 1B presents the contribution of these components as a function of the reaction cycles, where it can be seen that the integrated intensity of $\mathrm{Mn}^{3+}$ follows the opposite pattern from $\mathrm{Mn}^{2+}$. It is noticeable that during $\mathrm{N}_{2} \mathrm{O}$ catalytic decomposition the amount of $\mathrm{Mn}^{3+}$ increases, indicating that divalent manganese is oxidized. Under the chosen conditions $\left(350^{\circ} \mathrm{C}, 1 \mathrm{bar}\right.$, $10 \% \mathrm{NO}$ in $\mathrm{He}$ ), the $\mathrm{NO}$ treatments have only a small effect on the Mn valence. It is noticeable that the separation into $\mathrm{Mn}^{2+}$ and $\mathrm{Mn}^{3+}$ features and the separation of the pre-edge region from the edge jump can only be made thanks to the increased resolution with respect to normal XANES.

\subsection{STEM-EELS}

Additional information has been obtained with a scanning transmission electron microscope (STEM) to measure the electron energy loss spectrum (EELS). The EELS spectrum is providing similar information to that obtained by soft X-ray absorption. STEMEELS combines the spatial resolution of the electron microscope of $\sim 0.5 \mathrm{~nm}$ with the $\sim 0.5 \mathrm{eV}$ energy resolution of the EELS detector, resulting in a powerful tool that can characterize a single nanoparticle and provide chemical information about that particular chosen nanoparticle [7]. The discovery of TS-1 [8] opened a new trend in the preparation of microporous materials by incorporating different transitional metals in the structure of these materials. Although the influence of the incorporated metal in the catalytic reaction can be easily checked, the characterization of the metal sites and the location in the crystalline matrix is not so trivial. Here we present the case of Mn-ZSM-5 prepared using the hydrothermal synthesis method. Analysis shows X-ray diffraction patterns characteristic of the MFI structure, with around $95 \%$ of the present manganese in the trivalent state (as determined from XANES).

Fig. 2 presents a Scanning Electron Microscopy (SEM) picture of the prepared sample together with a chemical map obtained by measuring Si and Mn L edge of a 100x100 nm area of the surface crystal using STEM-EELS. The STEM-EELS area is indicated on the SEM image, where the crystal shown is only indicative and not exactly the same crystal in both images. The STEM-EELS chemical map show that the $\mathrm{Mn} / \mathrm{Si}$ ratio changes drastically through the same crystal. One observes a Mn-rich layer covering the Si-rich bulk of the crystal. The thickness of these Mn-rich layers is in the range of a few nanometers, which prevent their detection in X-ray diffraction patterns.

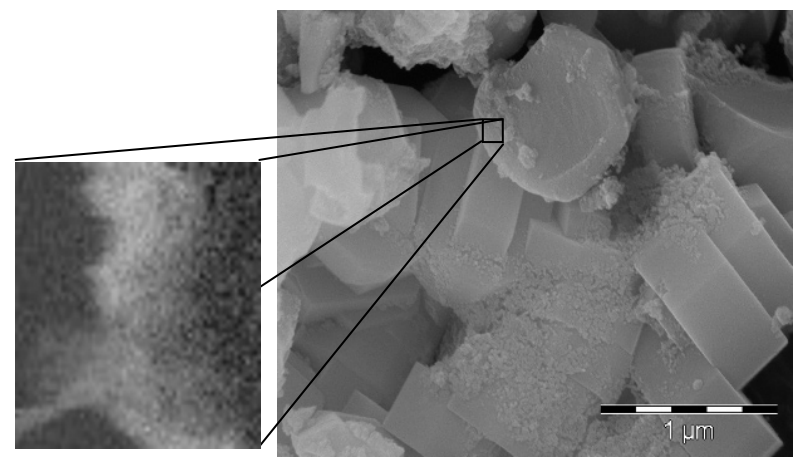

Fig. 2. SEM image $(2.5 \times 3 \mu \mathrm{m})$ of a hydrothermal prepared Mn-ZSM-5 and a small chemical map measured using STEM-EELS (100x100nm), (black-Manganese, white-Silicon) 


\subsection{X-MCD}

The X-MCD measurements were conducted in vacuum at $4 \mathrm{~K}$ using a $6 \mathrm{~T}$ superconducting magnet, in order to magnetize the paramagnetic centers. The soft X-ray $\mathrm{Mn}$ and $\mathrm{Fe} \mathrm{L}$ edges are measured with a resolution of $0.2 \mathrm{eV}$. X-MCD is an important characterization tool for paramagnetic sites in bio-inorganic chemistry [9]. The X-MCD signal is provided by all aligned magnetic moments in a certain system. Assuming a paramagnetic system of isolated moments, full magnetization can be obtained. In contrast a binuclear $\mathrm{Fe}$ or $\mathrm{Mn}$ center or large $\mathrm{Fe}$ and Mn clusters will couple anti-ferromagnetically, which thereby cancels its MCD effect. This implies that the magnitude of the X-MCD effect can be related to the amount of single Mn or Fe sites. In case of FeZSM5, it is found that the as-made sample contains only isolated Fe. Calcination lowers the isolated Fe sites to $70 \%$ and steaming reduced this number to $50 \%$.

\section{CONCLUSION}

The measurement of fluorescent $\mathrm{K} \alpha$ or $\mathrm{K} \beta$ transitions with a high-resolution spectrometer yields a range of new characterizations techniques, including HERFD-XANES. These highresolution spectra enabled a refined analysis of the pre-edge features, making it possible to separate contributions from species of different oxidation states during in-situ measurements as seen in the case of Mn-ZSM-5 system. This technique is readily applicable to all other $3 \mathrm{~d}$ transitions metal ions with loading even lower then $0.1 \mathrm{wt} \%$. STEM-EELS spectra have the advantage of their unrivalled spatial resolution of $0.5 \mathrm{~nm}$ combined with the possibility to accurately determine the elemental variation. A disadvantage of STEM-EELS is that the measurements require high vacuum conditions, although new developments can increase the pressure in the range of mbar. X-MCD is a direct tool to determine the amount of isolated metal sites in very low concentrations and can be applicable to all metals. Because these measurements are performed at $4 \mathrm{~K}$, this will limit the application to ex-situ conditions.

\section{REFERENCES}

[1] W. M. Heijboer; D. C. Koningsberger; B. M. Weckhuysen and F. M. F. de Groot, Catal. Today, $110(2005) 228$.

[2] D. C. Radu, H. J. Bitter, B. M. Weckhuysen and F. M. F. de Groot, in preparation.

[3] P. Glatzel, U. Bergmann, J. Yano, H. Visser, J. H. Robblee, W. W. Gu, F. M. F. de Groot, G. Christou, V. L. Pecoraro, S. P. Cramer and V. K. Yachandra, J. Am. Chem. Soc., 126 (2004) 9946.

[4] M. Wilke, F. Farges, P. E. Petit, G. E. Brown and F. Martin, Am. Mineral., 86 (2001) 714.

[5] W. M. Heijboer, P. Glatzel, K. R. Sawant, R. F. Lobo, U. Bergmann, R. A. Barrea, D. C. Koningsberger, B. M. Weckhuysen and F. M. F. de Groot, J. Phys. Chem. B, 108 (2004) 10002.

[6] F. M. F. de Groot, Chem. Rev., 101 (2001) 1779.

[7] O. Stephan, A. Gloter, D. Imhoff, M. Kociak, C. Mory, K. Suenaga, M. Tence and C. Colliex, Surf. Rev. Lett., 7 (2000) 475.

[8] M. Taramasso, G. Perego, B. Notari, US Patent No. 4410501 (1983).

[9] T. Funk, A. Deb, S. J. George, H. X. Wang and S. P. Cramer, Coord. Chem. Rev., 249 (2005) 3. 